\title{
Prognostic importance of apathy in syndromes associated with frontotemporal lobar degeneration
}

Claire J. Lansdall, PhD, Ian T.S. Coyle-Gilchrist, MBBS, Patricia Vázquez Rodríguez, MSc, Alicia Wilcox, MClin NeuroPsy, Eileen Wehmann, MPhil, Trevor W. Robbins, PhD, and James B. Rowe, BM, PhD Neurology ${ }^{\circledR}$ 2019;92:e1547-e1557. doi:10.1212/WNL.0000000000007249

\section{Correspondence}

Prof. Rowe

james.rowe@

mrc-cbu.cam.ac.uk

\begin{abstract}
Objective

To determine the influence of apathy, impulsivity, and behavioral change on survival in patients with frontotemporal dementia, progressive supranuclear palsy, and corticobasal syndrome.
\end{abstract}

\section{Methods}

We assessed 124 patients from the epidemiologic PiPPIN (Pick's Disease and Progressive Supranuclear Palsy, Prevalence and Incidence) study. Patients underwent detailed baseline cognitive and behavioral assessment focusing on apathy, impulsivity, and behavioral change. Logistic regression identified predictors of death within 2.5 years from assessment, including age, sex, diagnosis, cognition, and 8 neurobehavioral profiles derived from a principal component analysis of neuropsychological and behavioral measures.

\section{Results}

An apathetic neurobehavioral profile predicted death (Wald statistic $=8.119, p=0.004$, $\operatorname{Exp}(B)=2.912$, confidence interval $=>1[1.396-6.075])$ and was elevated in all patient groups. This profile represented apathy, weighted strongly to carer reports from the Apathy Evaluation Scale, Neuropsychiatric Inventory, and Cambridge Behavioral Inventory. Age at assessment, sex, and global cognitive impairment were not significant predictors. Differences in mortality risk across diagnostic groups were accounted for by their neuropsychiatric and behavioral features.

\section{Conclusions}

The relationship between apathy and survival highlights the need to develop more effective and targeted measurement tools to improve its recognition and facilitate treatment. The prognostic importance of apathy suggests that neurobehavioral features might be useful to predict survival and stratify patients for interventional trials. Effective symptomatic interventions targeting the neurobiology of apathy might ultimately also improve prognosis. 


\section{Glossary}

ALS = amyotrophic lateral sclerosis; bvFTD = behavioral variant frontotemporal dementia $\mathbf{C B S}=$ corticobasal syndrome; $d f=$ degrees of freedom; FTD = frontotemporal dementia; FTLD = frontotemporal lobar degeneration; PiPPIN = Pick's Disease and Progressive Supranuclear Palsy Prevalence and Incidence; PPA = primary progressive aphasia; PSP = progressive supranuclear palsy; svPPA = semantic variant primary progressive aphasia.

Dementia greatly increases mortality but the mechanisms of this effect are poorly understood. In the clinical syndromes associated with frontotemporal lobar degeneration (FTLD), there is substantial variation in survival rates. For example, survival is shortest for frontotemporal dementia (FTD) with motor neuron disease (typically 2-5 years), intermediate for progressive supranuclear palsy (PSP [Richardson syndrome]; typically 5-7 years), ${ }^{1,2}$ corticobasal syndrome (CBS; typically 6-8 years), ${ }^{3}$ and behavioral variant FTD (bvFTD; typically $5-8$ years $),{ }^{2}$ and longest in semantic variant primary progressive aphasia (svPPA; typically $10-12$ years $)^{2,3}$ or PSPparkinsonism (typically $10-12$ years). ${ }^{4}$ Despite improved knowledge of the clinical features and neuropathologic hallmarks of these FTLD syndromes, their influence on survival is unclear.

Predicting disease progression and patient trajectories is challenging, and the mechanisms underlying variations in survival rates remain elusive. Previous studies have reported only mild or null associations between survival and patient demographics, a positive family history, and dementia severity at the time of diagnosis, while highlighting the importance of neuropsychiatric and behavioral features. ${ }^{3,5,6}$ However, variations in estimated survival rates in part reflect the use of clinical $^{3,7}$ vs neuropathologic ${ }^{5,8}$ cohorts. Limited clinicopathologic correlations and inclusion of nonprogressive "phenocopy" patients may have affected previous estimates. Indeed, the removal of 24 such "phenocopy" cases from 91 clinical bvFTD cases reduced median survival from 9.0 to 7.6 years from onset. ${ }^{7}$

Improved clinical diagnostic criteria ${ }^{9}$ and assessment of the "spectrum" of FTLD ${ }^{10}$ present new opportunities to understand the determinants of poor survival, whether for disorders associated with primary tauopathies (PSP, CBS, nonfluent variant PPA, and half of bvFTD) or TDP-43 (TAR DNA-binding protein 43) pathologies (svPPA and half of bvFTD). Herein, we focus on the neuropsychiatric features that arise in many disorders of movement and cognition. For example, apathy has been associated with worse outcomes across a range of neurologic conditions including Alzheimer disease, stroke, ${ }^{11}$ Huntington disease, ${ }^{12}$ Parkinson disease, ${ }^{13}$ FTLD syndromes, ${ }^{14-16}$ and predementia states. ${ }^{17}$ Apathy correlates with poor functioning, caregiver distress, ${ }^{15}$ cognitive decline, ${ }^{13}$ increased dementia conversion rates, reduced quality of life, ${ }^{11}$ and poor prognosis. ${ }^{18}$ The association between apathy and survival may be causal, or reflect common influences of a third factor on both and disease-specific factors. However, a direct influence of apathy on survival warrants further investigation, not least because effective symptomatic treatments might also indirectly improve prognosis. ${ }^{6,19,20}$

We used the Pick's Disease and Progressive Supranuclear Palsy Prevalence and Incidence (PiPPIN) $)^{2}$ cohort to test the hypothesis that neurobehavioral components of FTLD syndromes are significant predictors of mortality. We used logistic regression to estimate the probability of death occurring within 2.5 years from assessment. Candidate predictor variables included age, sex, cognitive status, diagnosis, and the 8 neuropsychological principal components reported in reference 10. In view of previous studies highlighting the importance of apathy and related behavioral change over demographics, diagnosis, and cognitive status, we hypothesized that the neurobehavioral components strongly weighted toward assessment of apathy would be most influential on survival. Specifically, we predicted that carer-based estimates of apathy, everyday skills, and challenging behaviors would predict mortality, across the spectrum of disorders caused by FTLD.

\section{Methods}

\section{Standard protocol approvals, registrations, and patient consents}

The study was approved by the Cambridge 2 research ethics committee (reference 12/EE/0475) and supported by the National Institute for Health Research clinical research network (ID-15504). Informed consent was obtained at each study visit, with the personal consultee process used for participants who lacked mental capacity, in accordance with UK law. We anticipated approximately 150 patients based on prior epidemiologic estimates, which would provide good power $(>0.8)$ to detect small- to medium-sized group effects and correlations, and to identify $>4$ distinct neuropsychological components.

\section{Cohort}

Two hundred four patients were identified and recruited to the PiPPIN study, according to consensus clinical diagnostic criteria, ${ }^{2}$ plus 50 healthy age- and sex-matched controls. The occurrence and date of death were obtained from centralized United Kingdom National Health Service records. Exclusions for the current analysis included insufficient complete data for logistic regression (e.g., limited cognitive/functional, selfrated, carer-rated, or behavioral assessment), or assessment in 
the PiPPIN study too late for the follow-up interval at the time of analysis. Because logistic regression removes cases with missing data "list-wise" by default, the inclusion of multiple predictor variables (each of which may have a small but finite percentage of missing data) results in the additional exclusion of patients. Of the 204 PiPPIN patients, 124 undertook detailed neuropsychological assessments (PSP 35, CBS 29, PPA 33, bvFTD 27), of whom 112 had at least 30 months of follow-up at the time of analysis. The 50 healthy controls also undertook the neuropsychological assessment.

Participants were tested while on their usual medication: $40 \%$ took "antidepressant" medications (for affective or behavioral indications), 29\% dopaminergic medication, $4 \%$ antipsychotic medication, and $37 \%$ other centrally acting medications (benzodiazepines, antiepileptic, analgesics, pregabalin, or cholinesterase inhibitors).

\section{Statistical analysis}

All statistical analyses were performed using SPSS Statistics version 22 (IBM Corp., Armonk, NY). Kaplan-Meier survival curves were used to illustrate survival from assessment date and from estimated onset, by diagnostic group. Survival rates were compared by $\chi^{2}$ test. Logistic regression was used to identify significant predictors of death within 30 months from assessment. The principal outcome measure refers to 30 months because of similar sample sizes across groups (deceased $=50$ and alive $=62$ ). To predict survival at 30 months, patients were classified as "deceased" or "alive" using a cutoff of 913 days post assessment. Patients who were alive but had not yet lived 30 months from assessment were classified as "insufficient follow-up time" and excluded.

Logistic regression used the "Enter" method. Predictor variables included age at assessment, sex, cognitive status (Addenbrooke's Cognitive Examination-Revised), ${ }^{21}$ diagnosis (PSP, CBS, PPA, bvFTD), and the 8 principal behavioral components extracted from a neuropsychological and behavioral test battery of participants, and questionnaire responses of carers and clinician, using the principal components method. ${ }^{10}$ In brief, 22 questionnaires and behavioral measures assessing apathy, impulsivity, and related behavioral change were obtained, gaining insight from multiple perspectives including patient, carer, clinician, and objective tasks. The neurocognitive components revealed that (1) apathy and impulsivity are positively correlated, with validated measures of each loading onto the same components; (2) these behaviors are present in all syndromes associated with FTLD, to a varying degree; and (3) patient, carer, and objective measures differ, loading onto separable components and reflecting distinct neural correlates. ${ }^{10}$

Overall fit of the model was determined by the -2 loglikelihood and its associated $\chi^{2}$ statistic, using a threshold of $p<0.05$ indicating a significant fit of the data. Cox and Snell $R^{2}$ values provided an additional indication of model effect size. The influence of the independent variables on predicting outcome (death within 2.5 years) were determined by the significance of the Wald statistic $(p<0.05)$. Additional information regarding the directionality of effect was provided by the odds ratio $[(\operatorname{Exp}(B)]$; values $>1$ indicate increasing odds of outcome occurrence (death) with increased values of the predictor variable, while values $<1$ indicate decreasing odds of outcome occurrence with increased values of the predictor variable). Confidence intervals of the $\operatorname{Exp}(\mathrm{B})$ values were used to confirm the direction of the relationship in the population. Classification accuracy of the final model was compared to the baseline model (baseline model [constant only] $\%$ - new model [all predictor variables] \%) to determine whether inclusion of the independent variables resulted in significant model improvement. Residuals were also examined to assess model fit. Statistics included the following: standardized residuals to measure the model fit to the sample data ( $<1 \%$ of observations \pm 2.58$)$; Cook distance to measure the overall influence of an individual case on the model (values $<1$ ); and DFBeta statistics to measure the influence of a case on the values of $b$ (values $<1$ ).

Sensitivity (the percentage of cases that have the observed characteristic and were correctly predicted by the model [true positives]) and specificity (the percentage of cases that did not have the observed characteristics and were correctly predicted as not having it [true negatives]) of the model were also calculated (positive predictive value $=$ number of true positives/[number of true positives + number of false positives $]$; negative predictive value $=$ number of true negatives $/$ [number of true negatives + number of false negatives]).

\section{Data availability}

Anonymized data may be shared by request from a qualified investigator for noncommercial purposes, subject to participants' prior consent to data sharing.

\section{Results}

\section{Cohort, demographics, and clinical features}

Demographics and clinical features of the 124 patients and 50 healthy controls at baseline are detailed in table 1 . The patients included in the logistic regression analysis were similar to those who were not included, in terms of age, sex, and diagnostic group (table 2). The neurobehavioral profiles are summarized in table 3 , in terms of the loadings of each test in a principal component. The correlations of components with measures of cognition and function are reported in table 4.

At 30 months post assessment, 50 patients had died, including 21 PSP, 15 CBS, 5 PPA, and 9 bvFTD. The remaining patients were classified as "alive" $(\mathrm{n}=62)$ or were excluded because of insufficient follow-up time $(n=12)$. Twenty-four cases were removed list-wise during the logistic regression because of missing data of interest. The final logistic regression subset was representative of the full cohort from 
Table 1 Demographics and disease characteristics by diagnostic group at baseline

\begin{tabular}{|c|c|c|c|c|c|c|c|c|}
\hline Variable & Controls & $\begin{array}{l}\text { All } \\
\text { patients }\end{array}$ & $\begin{array}{l}\text { Control vs } \\
\text { patient } \\
\text { difference }^{a}\end{array}$ & PSP & CBS & PPA & bvFTD & $\begin{array}{l}\text { Patient group } \\
\text { difference }\end{array}$ \\
\hline No. & 50 & 124 & - & 35 & 29 & 33 & 27 & - \\
\hline Age at assessment, y & $70.6 \pm 6.5$ & $69.3 \pm 8.4$ & NS & $72.2 \pm 8.5$ & $68.9 \pm 8.3$ & $71.5 \pm 6.6$ & $63.5 \pm 7.9$ & $a, d, f, g$ \\
\hline Symptom duration, y & NA & $4.7 \pm 2.9$ & - & $4.5 \pm 3.5$ & $4.5 \pm 2.1$ & $4.9 \pm 2.8$ & $5.1 \pm 3.1$ & NS \\
\hline Sex, M/F & $27 / 23$ & $62 / 62$ & NS & $19 / 16$ & $13 / 16$ & $15 / 18$ & $15 / 12$ & NS \\
\hline $\begin{array}{l}\text { Addenbrooke's Cognitive } \\
\text { Examination-Revised }(/ 100)^{21}\end{array}$ & $95.6 \pm 4.4$ & $64.1 \pm 22.4$ & $p<0.001$ & $75.5 \pm 14.6$ & $65.7 \pm 21.3$ & $53.1 \pm 21.6$ & $59.0 \pm 26.9$ & $a, c, d, e$ \\
\hline $\begin{array}{l}\text { Mini-Mental State } \\
\text { Examination }(/ 30)\end{array}$ & $29.3 \pm 1.2$ & $22.2 \pm 6.8$ & $p<0.001$ & $25.0 \pm 4.8$ & $22.0 \pm 6.6$ & $19.4 \pm 7.6$ & $21.4 \pm 7.6$ & $a, c$ \\
\hline $\begin{array}{l}\text { Frontal Assessment } \\
\text { Battery }(/ 18)^{40}\end{array}$ & $16.8 \pm 1.2$ & $9.8 \pm 4.4$ & $p<0.001$ & $10.5 \pm 4.0$ & $10.0 \pm 4.4$ & $9.3 \pm 4.1$ & $9.4 \pm 5.3$ & NS \\
\hline $\begin{array}{l}\text { Cambridge Behavioral } \\
\text { Inventory }(/ 180)^{24}\end{array}$ & $5.2 \pm 5.6$ & $67.2 \pm 35.0$ & $p<0.001$ & $56.0 \pm 32.4$ & $73.5 \pm 30.6$ & $57.1 \pm 41.1$ & $85.6 \pm 26.2$ & $a, d, g$ \\
\hline
\end{tabular}

Abbreviations: bvFTD = behavioral variant frontotemporal dementia; $C B S=$ corticobasal syndrome; NA = not applicable; NS = not significant; PPA = primary progressive aphasia; PSP = progressive supranuclear palsy.

${ }^{a}$ Group difference between controls and all patients was assessed by Student $t$ test, corrected for multiple comparisons. Group differences between patient groups were assessed by analysis of variance: $a=$ significant F-contrast across 4 patient groups $(p<0.05)$, b-g pairwise t-contrasts using post hoc least significant difference correction for multiple comparisons $(p<0.05)$ : $b=$ PSP vs CBS; $c=$ PSP vs PPA; $d=P S P$ vs bvFTD; $=$ = CBS vs PPA; $f=C B S$ vs bvFTD; $g=$ PPA vs bvFTD.

which the neurobehavioral profiles were derived, ${ }^{10}$ in terms of age, sex, and diagnosis (table 2).

Survival ranged from 22 to 910 days after assessment (PSP 64-881 days, CBS 22-791 days, PPA 308-910 days, bvFTD 261-761 days). Of the 124 patients, 42 had died at 24 months, including 20 PSP, 11 CBS, 3 PPA, and 8 bvFTD, 81 were alive, and one had insufficient follow-up time. At 36 months, 55 patients had died including 22 PSP, $18 \mathrm{CBS}, 6 \mathrm{PPA}$, and 9 bvFTD, 39 were alive, and 30 had insufficient follow-up time.

\section{Logistic regression}

Including all predictors resulted in a significant fit to the model $\left(-2 \log\right.$ likelihood $=88.401, \chi^{2}=29.9$, degrees of freedom $[d f]=14, p=0.008$, Cox and Snell $R^{2}=0.288$, Nagelkerke $\left.R^{2}=0.390\right)$. Model classification accuracy improved from $60.2 \%$ at baseline (including only a constant) to $73.9 \%$ following inclusion of the predictor variables. The model correctly classified 43 as alive while incorrectly classifying 13, and correctly classified 22 as dead while incorrectly classifying an additional 10, resulting in a positive predictive value of $81 \%$ and negative predictive value of $63 \%$ ( positive predictive value $=43 / 43+10=0.811$, negative predictive value $=22 / 22+13=0.629$ ) .

Of the predictor variables, carer-rated change in everyday skills, self-care, and apathy (component 2) were the most significant predictor of death within 2.5 years from PiPPIN assessment (Wald statistic $=8.119, p=0.004, \operatorname{Exp}(B)=2.912$, confidence interval $=>1$ [1.396-6.075]; table 5). An $\operatorname{Exp}(B)$ value $>1$ (and confidence intervals both $>1$ ) indicated that increases in component 2 (weighted toward the carer-rated Apathy Evaluation Scale, ${ }^{22}$ Neuropsychiatric Inventory apathy subscore, ${ }^{23}$ and Cambridge Behavioral Inventory ${ }^{24}$ subscores of everyday skills, self-care, sleep, and motivation) significantly increased the odds of death within the 2.5-year time period. All patient groups scored significantly higher than controls on component 2 (figure 1).

Table 2 Comparison between patients included in the logistic regression cohort and those excluded

\begin{tabular}{|c|c|c|c|c|}
\hline & Excluded $(n=61)$ & Included $(n=88)$ & Statistic ${ }^{a}$ & $p$ Value \\
\hline Age & $69.0 \pm 8.6$ & $69.7 \pm 8.5$ & 0.47 & 0.64 \\
\hline Sex & M 36; F 25 & M 40; F 48 & 2.65 & 0.10 \\
\hline Diagnosis & PSP 13, CBS 13, PPA 18, bvFTD 17 & PSP 28, CBS 21, PPA 23, bvFTD 16 & 3.22 & 0.36 \\
\hline
\end{tabular}

Abbreviations: bvFTD = behavioral variant frontotemporal dementia; CBS = corticobasal syndrome; PPA = primary progressive aphasia; PSP = progressive supranuclear palsy.

a Statistic: age = Student $t$ test ( $t$ statistic $p>0.05)$; sex $=\chi^{2}$ test $\left(\chi^{2} p>0.05\right)$; diagnosis = analysis of variance $(F$ statistic, $p>0.05)$. 


\begin{tabular}{|c|c|c|c|c|c|c|c|c|}
\hline Component & 1 & 2 & 3 & 4 & 5 & 6 & 7 & 8 \\
\hline Name & $\begin{array}{l}\text { Patient-rated } \\
\text { change }\end{array}$ & $\begin{array}{l}\text { Carer-rated everyday skills and } \\
\text { self-care }\end{array}$ & $\begin{array}{l}\text { Carer-rated challenging } \\
\text { behaviors }\end{array}$ & $\begin{array}{l}\text { Impulsive } \\
\text { behaviors }\end{array}$ & $\begin{array}{l}\text { Impulsivity self- } \\
\text { report }\end{array}$ & $\begin{array}{l}\text { Goal-directed decision- } \\
\text { making }\end{array}$ & $\begin{array}{l}\text { Stop Signal } \\
\text { Task }\end{array}$ & $\begin{array}{l}\text { Outcome } \\
\text { sensitivity }\end{array}$ \\
\hline Eigenvalue I/R & $4.963 / 3.438$ & $2.183 / 2.284$ & $1.664 / 2.145$ & $1.514 / 1.819$ & $1.385 / 1.640$ & $1.186 / 1.284$ & $1.111 / 1.245$ & $1.039 / 1.188$ \\
\hline \multicolumn{9}{|l|}{ Questionnaires } \\
\hline AES 1 & $0.832^{\mathrm{a}}$ & -0.069 & -0.121 & 0.151 & -0.078 & -0.003 & -0.041 & -0.069 \\
\hline BIS 1 & $0.735^{\mathrm{a}}$ & 0.086 & 0.083 & 0.221 & 0.080 & -0.003 & -0.095 & -0.052 \\
\hline BDI-T & $0.756^{a}$ & 0.345 & 0.100 & 0.073 & 0.158 & 0.097 & -0.026 & -0.030 \\
\hline MEI-T & $-0.837^{a}$ & -0.232 & -0.061 & -0.109 & -0.023 & 0.034 & 0.142 & 0.007 \\
\hline SHAPS-T & $0.688^{a}$ & 0.147 & 0.281 & -0.067 & -0.276 & -0.136 & 0.068 & 0.075 \\
\hline AES 2 & 0.067 & $0.714^{a}$ & $0.529^{a}$ & 0.074 & 0.035 & 0.006 & -0.110 & -0.151 \\
\hline CBI 1 & 0.035 & 0.118 & $0.880^{a}$ & 0.078 & 0.104 & -0.135 & -0.066 & -0.069 \\
\hline CBI 2 & 0.233 & $0.831^{a}$ & -0.084 & 0.151 & -0.113 & 0.023 & -0.155 & 0.042 \\
\hline NPI-A & 0.192 & $0.705^{a}$ & 0.355 & 0.119 & -0.086 & 0.048 & 0.029 & -0.050 \\
\hline NPI-D & 0.135 & 0.083 & $0.825^{a}$ & -0.008 & -0.017 & 0.039 & 0.017 & 0.092 \\
\hline BIS 2 & 0.022 & -0.121 & -0.015 & -0.100 & $0.841^{a}$ & -0.023 & -0.065 & 0.077 \\
\hline BIS/BAS 1 & -0.198 & -0.005 & 0.265 & 0.083 & $0.631^{a}$ & 0.375 & -0.209 & -0.011 \\
\hline BIS/BAS 2 & 0.068 & 0.090 & -0.088 & 0.042 & 0.242 & -0.179 & 0.141 & $0.804^{a}$ \\
\hline \multicolumn{9}{|l|}{$\begin{array}{l}\text { Performance- } \\
\text { based }\end{array}$} \\
\hline IST 1 & -0.188 & -0.204 & -0.080 & -0.177 & 0.013 & $0.556^{a}$ & 0.311 & 0.052 \\
\hline IST 2 & 0.170 & 0.030 & -0.037 & $0.683^{a}$ & -0.128 & 0.365 & -0.166 & 0.006 \\
\hline IST 3 & 0.255 & 0.382 & -0.198 & -0.167 & 0.335 & -0.007 & 0.283 & -0.001 \\
\hline CRRT 1 & 0.007 & 0.014 & -0.006 & $0.658^{a}$ & -0.013 & -0.104 & 0.390 & 0.109 \\
\hline CRRT 2 & 0.084 & 0.162 & -0.037 & 0.063 & 0.078 & $0.725^{a}$ & -0.031 & -0.078 \\
\hline Go/NoGo & -0.259 & -0.135 & -0.113 & $-0.642^{\mathrm{a}}$ & 0.130 & 0.042 & 0.259 & 0.007 \\
\hline Saccade & -0.162 & -0.198 & -0.081 & $-0.530^{a}$ & -0.319 & 0.221 & 0.018 & 0.158 \\
\hline SST 1 & 0.183 & 0.109 & 0.021 & 0.044 & 0.167 & -0.087 & $-0.793^{a}$ & 0.030 \\
\hline & & & & & & & & Contir \\
\hline
\end{tabular}




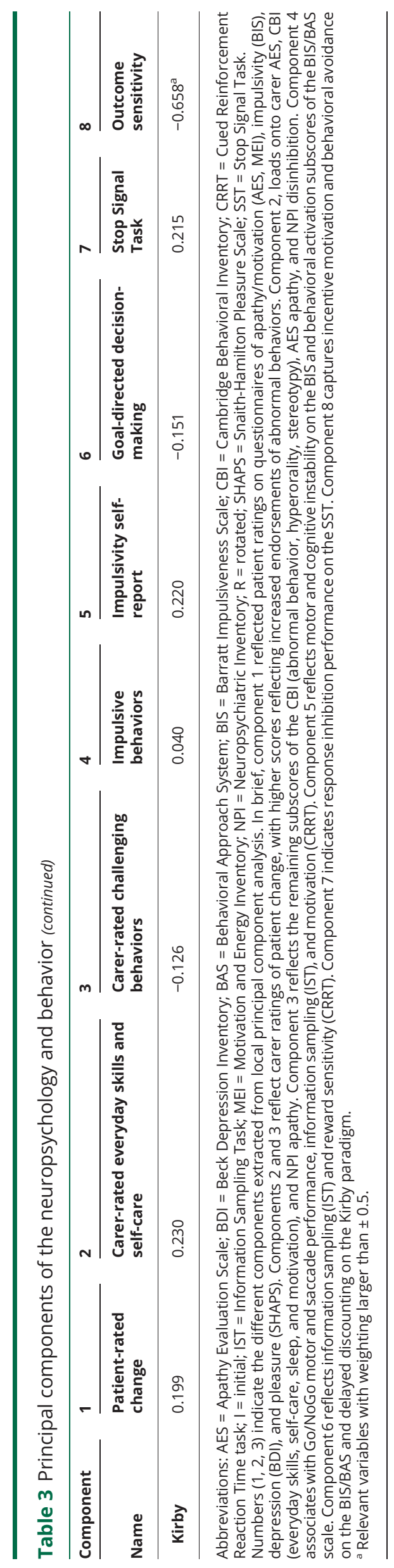

Component 8, reflecting insensitivity to reward on the Kirby and behavioral inhibition on the Barratt Impulsiveness Scale/ Behavioral Activation System, was marginal. The effect of age at assessment did not reach significance. Examination of the residuals confirmed good model fit: standardized residuals were all within \pm 2.58 , Cook distance, and DFBeta values were $<1$.

\section{Kaplan-Meier survival analysis}

Survival rates from PiPPIN assessment were significantly different depending on the diagnostic group (figure 2). Kaplan-Meier survival curves showed significant differences in survival from PiPPIN assessment (figure 2A; log-rank [Mantel-Cox] $\chi^{2}=20.9, d f=3, p<0.001$; pairwise comparisons revealed significant differences for PSP vs PPA $\chi^{2}=17.0$, $p<0.001$; PSP vs bvFTD $\chi^{2}=6.0, p<0.05$; CBS vs PPA $\chi^{2}=$ 14.0, $p<0.001$; and CBS vs bvFTD $\left.\chi^{2}=4.6, p<0.05\right)$ and from onset (figure 2B; log-rank [Mantel-Cox] $\chi^{2}=18.0, d f=$ $3, p<0.001$, pairwise comparisons revealed significant differences for PSP vs PPA $\chi^{2}=11.2, p=0.001$; PSP vs bvFTD $\chi^{2}=4.8, p<0.05$; CBS vs PPA $\chi^{2}=13.5, p<0.001$; CBS vs bvFTD $\left.\chi^{2}=5.7, p<0.05\right)$. Date from onset was missing for 3 participants, hence the reduced sample size for figure $2 \mathrm{~B}$.

\section{Discussion}

This study confirms the significance of apathy for survival, across the major syndromes associated with FTLD, including behavioral and language variants of FTD, PSP, and CBS. The results stem from a cross-sectional epidemiologic cohort that included community-based as well as specialist-center recruitment of prevalent cases, diagnosed according to current consensus diagnostic criteria. The carers' rating of apathy and functional decline (including self-care and motivation) was the most significant predictor of death within 30 months, even after adjusting for diagnostic group. The relevant apathy profile was weighted toward the Apathy Evaluation Scale, Neuropsychiatric Inventory, and Cambridge Behavioral Inventory subscores (principal component 2 , table 3 ). The patients' age and general cognitive ability did not predict survival. In just 2.5 years in the cross-sectional cohort, 50 of 24 patients had died, in keeping with typical survival rates in previous studies of each disease. Despite differences in survival across groups (shortest for PSP, followed by CBS, bvFTD, and PPA), the diagnostic group did not predict death when apathy and other neurobehavioral profiles were included in the model.

These findings emphasize the prognostic importance of behavioral change in FTLD syndromes, over and above demographic features and diagnostic classification. ${ }^{6,20,25}$ This effect includes patients with diagnoses that are not defined by the presence of behavioral or personality change. One study classified patients with FTLD into specific phenotypes using latent profile analysis of neuropsychological, functional, and behavioral data. ${ }^{26}$ The prognosis was significantly worse in the "pseudomanic" group, who exhibited greater disinhibition 
Table 4 Correlations of components with measures of cognition and function

\begin{tabular}{llllllll}
\hline Variable & PC1 & PC2 & PC3 & PC4 & PC5 & PC6 & PC7 \\
\hline PSPRS & 0.134 & $0.550^{\mathrm{a}}$ & -0.224 & $-0.281^{\mathrm{b}}$ & 0.078 & 0.074 & -0.17 \\
\hline FRS\% & -0.01 & $-0.531^{\mathrm{a}}$ & $-0.450^{\mathrm{a}}$ & $0.229^{\mathrm{b}}$ & 0.16 & -0.047 & -0.095 \\
\hline ACE-R & 0.199 & -0.082 & -0.191 & -0.083 & 0.199 & 0.101 & 0.13 \\
\hline MMSE & 0.153 & -0.143 & -0.187 & -0.058 & 0.189 & 0.061 & -0.066 \\
\hline FAB & 0.021 & -0.051 & -0.094 & -0.121 & 0.087 & -0.021 & 0.146 \\
\hline CBI & -0.1 & $0.496^{\mathrm{a}}$ & $0.546^{\mathrm{a}}$ & $-0.187^{\mathrm{b}}$ & -0.087 & -0.063 & -0.038 \\
\hline
\end{tabular}

Abbreviations: $A C E-R=$ Addenbrooke's Cognitive Examination-Revised; $C B I=$ Cambridge Behavioral Inventory; FAB = Frontal assessment battery; FRS\% = centile on Frontotemporal Dementia Rating Scale; MMSE = Mini Mental State Examination; PC = principal component; PSPRS = PSP Rating Scale.

PC1-8 refer to the components described in table 3. Pearson correlations thresholded at:

a $p<0.001$ or

${ }^{\mathrm{b}} p<0.05$.

and abnormal social conduct. ${ }^{6}$ It is of interest that their "pseudo-depressed" group had a better prognosis over time, ${ }^{6}$ despite the potential confusion between depression and apathy. Consistent with such studies, we suggest that apathy, but not depression, is most associated with poor outcomes and functional decline, reflecting the distinct underlying neurobiology of apathy and depression. ${ }^{11}$
Impulsive and challenging social behaviors (component 3) were not a significant predictor of reduced survival, although they may increase health care costs and carer burden. The importance of apathy, rather than disinhibition, in causing functional impairment and disability has been reported in bvFTD, perhaps because isolated disinhibition is unusual in bvFTD. ${ }^{16}$ Our study extends this result to other syndromes associated with FTLD.

Table 5 Logistic regression of the influence of predictor variables on death 2.5 years post PiPPIN assessment

\begin{tabular}{|c|c|c|c|c|c|c|}
\hline \multirow[b]{2}{*}{ Variables } & \multirow[b]{2}{*}{ Wald statistic } & \multirow[b]{2}{*}{ Degrees of freedom } & \multirow[b]{2}{*}{$\operatorname{Exp}(B)$} & \multirow[b]{2}{*}{ Significance } & \multicolumn{2}{|c|}{$95 \% \mathrm{Cl}$ for $\operatorname{Exp}(\mathrm{B})$} \\
\hline & & & & & Lower & Upper \\
\hline ACE-R total score & 0.095 & 1 & 0.995 & 0.758 & 0.967 & 1.025 \\
\hline Age at assessment & 3.601 & 1 & 1.077 & 0.058 & 0.998 & 1.162 \\
\hline Sex & 0.027 & 1 & 1.096 & 0.871 & 0.365 & 3.293 \\
\hline Diagnosis Collapsed & 1.855 & 3 & - & 0.603 & - & - \\
\hline Diagnosis 1 & 1.080 & 1 & 1.710 & 0.299 & 0.622 & 4.705 \\
\hline Diagnosis 2 & 0.012 & 1 & 0.940 & 0.912 & 0.312 & 2.828 \\
\hline Diagnosis 3 & 1.274 & 1 & 0.518 & 0.259 & 0.165 & 1.625 \\
\hline PC1 & 2.913 & 1 & 1.646 & 0.088 & 0.929 & 2.919 \\
\hline PC2 & 8.119 & 1 & 2.912 & $0.004^{a}$ & 1.396 & 6.075 \\
\hline PC3 & 1.849 & 1 & 1.474 & 0.174 & 0.843 & 2.580 \\
\hline PC4 & 0.093 & 1 & 0.880 & 0.760 & 0.386 & 2.006 \\
\hline PC5 & 1.188 & 1 & 1.290 & 0.276 & 0.816 & 2.038 \\
\hline PC6 & 1.572 & 1 & 1.377 & 0.210 & 0.835 & 2.272 \\
\hline PC7 & 2.653 & 1 & 0.672 & 0.103 & 0.416 & 1.084 \\
\hline PC8 & $4.354^{\mathrm{a}}$ & $1^{a}$ & $0.509^{a}$ & $0.037^{b}$ & 0.270 & 0.960 \\
\hline Constant & 4.321 & 1 & 0.002 & 0.038 & - & - \\
\hline \multicolumn{7}{|c|}{$\begin{array}{l}\text { Abbreviations: } A C E-R=\text { Addenbrooke's Cognitive Examination-Revised; } C l=\text { confidence interval; PC = principal component; PiPPIN }=\text { Pick's Disease and } \\
\text { Progressive Supranuclear Palsy Prevalence and Incidence. } \\
\text { Degrees of freedom is equal to the number of parameters in the model. Significant predictors of death } 2.5 \text { years ( } 30 \text { months) post PiPPIN assessment: } \\
\text { a } p<0.01 \text {. } \\
\text { b } p<0.05 \text {. }\end{array}$} \\
\hline
\end{tabular}




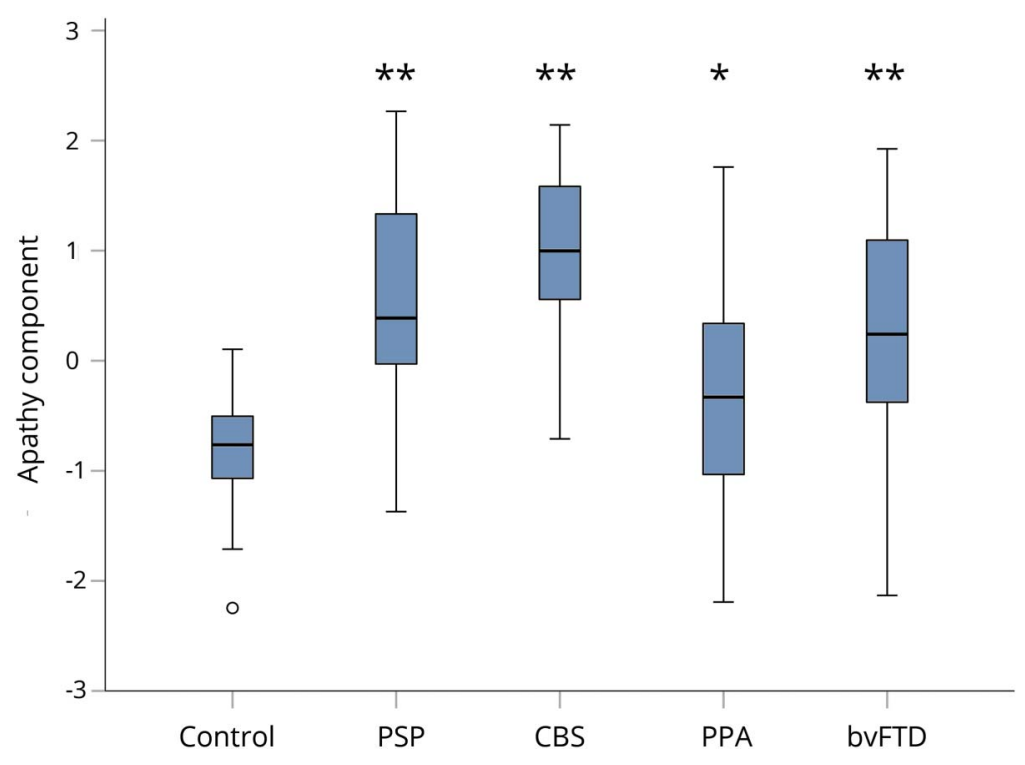

Patients scored significantly higher than controls on component 2 across diagnostic groups (analysis of variance, least significant difference correction for multiple comparisons, control vs PSP, CBS, bvFTD ** $p<0.001$, vs PPA * $p<0.05$ ). Component 2 had strong loadings from the carer-rated Apathy Evaluation Scale, Neuropsychiatric Inventory apathy subscore, and Cambridge Behavioral Inventory subscores of everyday skills, self-care, sleep, and motivation. Overall, higher scores on this neurobehavioral component reflected increased endorsement of apathy. bvFTD = behavioral variant frontotemporal dementia; CBS = corticobasal syndrome; PPA = primary progressive aphasia; $\mathrm{PSP}=$ progressive supranuclear palsy.
The influence of behavioral changes on survival is further emphasized by the observation that patients with comorbid FTD-ALS (amyotrophic lateral sclerosis) survive up to a year less than patients with "motor only" symptoms. Apathy is common in ALS, affecting $40 \%$ to $80 \%$ of patients, ${ }^{18}$ and may precede motor symptoms. In the context of ALS, apathy is also an independent, negative prognostic factor, significantly predicting survival even after controlling for clinical factors and symptom duration: median survival of patients with ALS who have moderate to severe apathy was significantly shorter than those with mild apathy or no apathy (21.7 vs 49.9 vs 51.9 months, respectively). ${ }^{18}$

We have previously used voxel-based morphometry and diffusion tensor imaging to examine the structural brain changes associated with apathy and impulsivity, revealing marked white matter atrophy of the brainstem and widespread gray matter changes in thalamus, striatum, and cortical connections. ${ }^{10,27}$ Patients with PSP and CBS scored highly on symptoms associated with brainstem and midbrain atrophy. Prominent bulbar symptoms, reflecting brainstem pathology, increase the likelihood of death, in part by choking and aspiration. ${ }^{28}$ However, frontal atrophy has also been linked to poor outcomes in CBS, ${ }^{20}$ PSP, and FTD. ${ }^{3}$ Across FTLD, the "pseudomanic" phenotype classified in reference 6 (2009) with reduced survival demonstrated worse hypoperfusion of the frontal cortex. Worsening fronto-subcortical pathology, linked to behavioral changes, also predicts greater mortality in patients with autopsy-proven corticobasal degeneration and FTD. ${ }^{9,20}$

The lack of a significant influence of global cognitive status on survival is perhaps surprising, but accords with previous observations that behavioral change rather than cognitive decline is a marker of progression and prognosis in syndromes associated with FTLD. ${ }^{6,25}$ The relationship between apathy and cognition is complex: apathy might cause cognitive worsening but it may also be a consequence of cognitive decline. In predementia states, apathetic patients consistently show more rapid cognitive and functional decline and increased dementia conversion rates compared to nonapathetic groups. ${ }^{17}$ In Parkinson disease, apathetic patients converted to dementia more frequently than nonapathetic patients, in 18 months. ${ }^{13}$ Even in those who did not develop dementia, apathetic groups showed significantly greater cognitive decline, specifically in terms of executive function deficits in response inhibition and action initiation, emphasizing the link between cognition and apathy. In mild cognitive impairment and early Alzheimer disease, patients with apathy had an approximately 8-fold-greater risk of conversion to dementia over 3 years, even after controlling for potential confounds including age, sex, education, and episodic memory performance. ${ }^{29}$

This link between apathy, dementia, and survival, raises the hypothesis that successful symptomatic treatment may alter patient trajectories, including perhaps survival. Testing this hypothesis will require development of more effective symptomatic treatments targeting the underlying causes of apathy. Such an effect would challenge the long-standing dichotomy between symptomatic and disease-modifying therapies in FTLD.

The diagnostic group was not a significant predictor of survival in the presence of neurobehavioral profiles, despite significant differences in survival across groups if considered alone. This suggests that simple categorical diagnostic grouping (using clinical diagnostic criteria for the major syndromes associated with FTLD) is of limited value for 


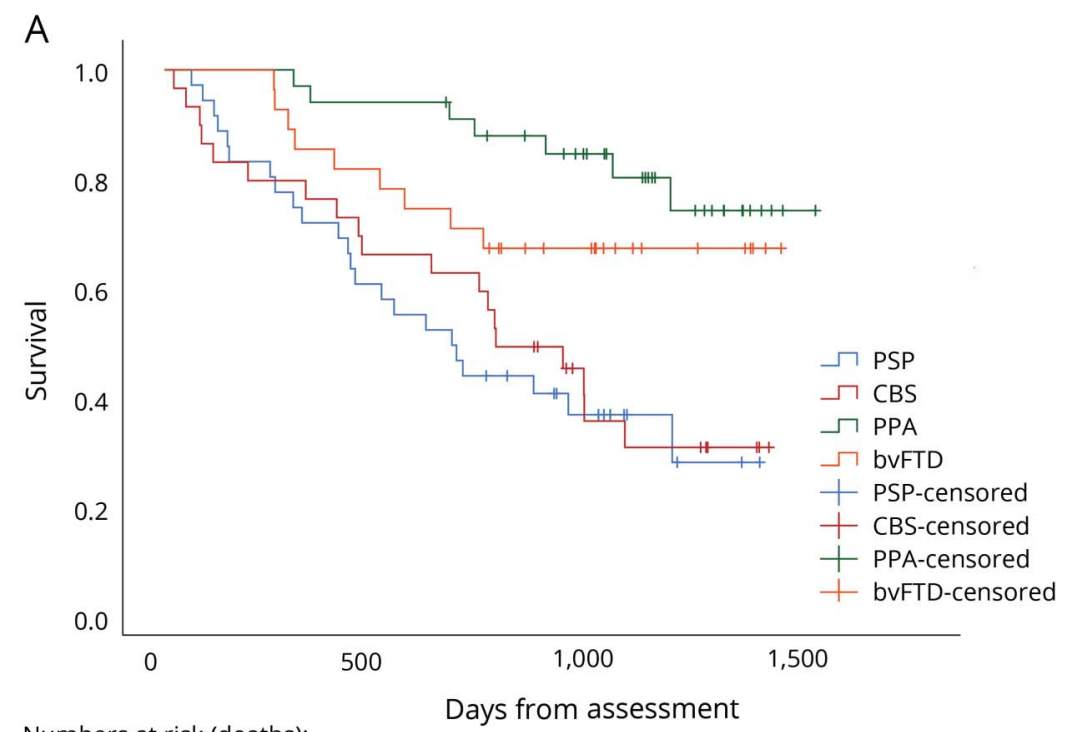

\begin{tabular}{lrrrrrrrr}
\multicolumn{7}{l}{ Numbers at risk (deaths): } \\
Total & 124 & $(31)$ & 93 & $(21)$ & 54 & $(6)$ & 1 & $(0)$ \\
PSP & 35 & $(14)$ & 21 & $(8)$ & 9 & $(1)$ & 0 & $(0)$ \\
CBS & 29 & $(10)$ & 19 & $(6)$ & 9 & $(3)$ & 0 & $(0)$ \\
PPA & 33 & $(2)$ & 31 & $(3)$ & 23 & $(2)$ & 1 & $(0)$ \\
bVFTD & 27 & $(5)$ & 22 & $(4)$ & 13 & $(0)$ & 0 & $(0)$
\end{tabular}

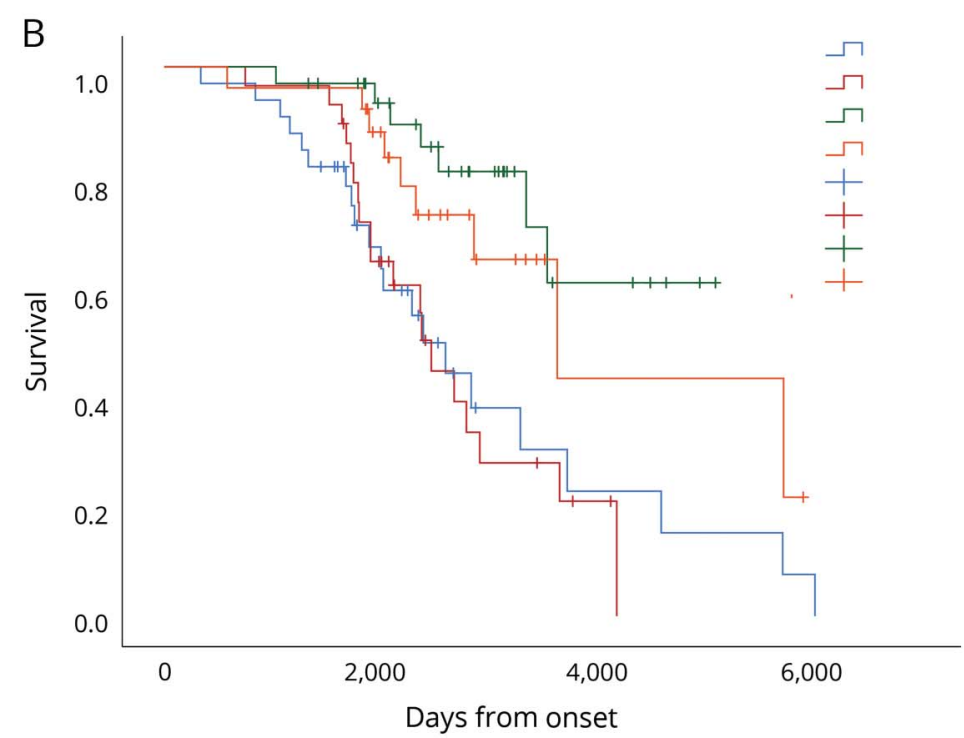

Numbers at risk (deaths):

$\begin{array}{lrrrrrrrr}\text { Total } & 121 & (25) & 79 & (26) & 12 & (4) & 2 & (1) \\ \text { PSP } & 33 & (10) & 17 & (8) & 3 & (2) & 1 & (1) \\ \text { CBS } & 29 & (10) & 17 & (8) & 2 & (1) & 1 & (0) \\ \text { PPA } & 33 & (2) & 25 & (5) & 5 & (0) & 0 & (0) \\ \text { bvFTD } & 26 & (3) & 20 & (5) & 2 & (1) & 0 & (0)\end{array}$

\begin{abstract}
Survival rates differed significantly between diagnostic groups from PiPPIN assessment (A: log-rank [Mantel-Cox] $\chi^{2}=20.9, d f=3, p<0.001$; pairwise comparisons revealed significant differences for PSP vs PPA $\chi^{2}=17.0, p<0.001$ PSP vs bvFTD $\chi^{2}=6.0, p<0.05$; CBS vs PPA $\chi^{2}=14.0, p<$ 0.001 ; and CBS vs bvFTD $\left.\chi^{2}=4.6, p<0.05\right)$, and from onset (B: log-rank [Mantel-Cox] $\chi^{2}=18.0, d f=3, p<0.001$; pairwise comparisons revealed significant differences for PSP vs PPA $\chi^{2}=11.2, p=0.001$; PSP vs bvFTD $\chi^{2}=4.8, p<$ 0.05 ; CBS vs PPA $\chi^{2}=13.5, p<0.001$; CBS vs bvFTD $\chi^{2}=5.7$ $p<0.05)$. bvFTD $=$ behavioral variant frontotemporal dementia; CBS = corticobasal syndrome; $d f=$ degrees of freedom; PiPPIN = Pick's Disease and Progressive Supranuclear Palsy Prevalence and Incidence; PPA = primary progressive aphasia; PSP = progressive supranuclear palsy.
\end{abstract}

prognostication. ${ }^{6}$ If observations are available that capture behavioral change in these disorders, such as apathy, then these are better able to predict survival than diagnosis alone. While neuropathologic studies have led to increased fractionation of FTLD syndromes, the influence of distinct pathologies on survival remain unclear, with both tau-positive and tau-negative cases correlating with reduced survival. ${ }^{3,5,8,30,31} \mathrm{Al}$ though variations in the survival rates across clinical phenotypes have been reported, in the presence of wide phenotypic variation, the diagnosis alone does not appear to be strongly predictive of survival. ${ }^{6}$

We argue instead that the presence and severity of apathy across the spectrum of FTLD disorders influences survival, while the remaining features that underlie diagnosis (but are not captured by the component) do not significantly influence prognosis. In other words, the differences in prognosis between syndromes (figure 2) are driven by the phenotypic 
features or their neurobiological correlates that are present across multiple disorders, albeit to a variable degree. This places the emphasis on the domains of impairment, akin to the Research Domain Criteria of neuropsychiatric disorders. ${ }^{32}$ In bvFTD, for example, current criteria require 3 of 6 core features. Therefore, 2 patients might have no clinical overlap, such that the presence of a clinical feature rather than the diagnosis per se determines prognosis. We also group the patients with PPA into a single group for analysis, in keeping with the high-level division of FTD into behavioral and language variants. This brings cases with marked clinical heterogeneity of svPPA, nonfluent variant PPA, and logopenic PPA into a single group. However, it has the advantage of approximating group sizes, while permitted phenotypic expression between subgroups to be retained in terms of the individuals' loading value on each of the neurobehavioral components.

The observation that the diagnosis category is not predictive of survival provides additional support for the transdiagnostic approach adopted by the PiPPIN study ${ }^{2,10}$ and has direct implications for the design of future clinical investigations. For a clinical trial of symptomatic treatments, we propose that emphasis should be placed on recruiting patients who present with that symptom (e.g., apathy) rather than on patients defined by a diagnostic label (e.g., bvFTD). In PiPPIN, apathy (component 2) was abnormal across the diagnostic groups (figure 1). Profound apathy and associated behavioral changes are increasingly recognized in PSP and $\mathrm{CBS}^{33-37}$ despite being largely overlooked because of predominant motor impairments.

Of note, it remains unclear whether the relationship between apathy and mortality is causal or merely correlational. Apathy may accelerate rapid decline to death or may represent a marker of other underlying factors that correlate with both apathy and survival, such as brainstem degeneration (the neural correlate of component $2^{10}$ ). Here, we do not have evidence of causality, but the relationship between apathy and survival raises the possibility that treating apathy would improve outcomes. Interventional studies are required, to either treat apathy at the behavioral level (symptomatic) or to target the underlying neural correlates (disease-modifying). However, it is likely that apathy in the context of syndromes associated with FTLD is multifaceted. For example, gray matter atrophy and white matter degeneration ${ }^{10,27}$ are accompanied by changes in noradrenaline, dopamine, and serotonin that modulate motivation, attention, and reinforcement learning. ${ }^{38}$ The quantification of these deficits, and the resulting neurobehavioral profiles such as apathy, may enable more effective stratification of patients for clinical trials.

A number of limitations should be acknowledged. Inherent to most clinical studies estimating survival in FTLD syndromes is the variability of clinicopathologic correlations, with potential misdiagnosis and thereby inaccurate within-syndrome estimates. Semantic dementia and PSP-Richardson syndrome have the highest accuracy (>90\%), but for CBS, only approximately $60 \%$ have corticobasal degeneration. ${ }^{39}$ However, with the possible exception of logopenic PPA, those with a misdiagnosis often have another FTLD pathology, further emphasizing the benefits of a transdiagnostic approach. Here, we included collapsed diagnostic groups of PSP, CBS, bvFTD, and PPA in the logistic regression and acknowledge that the clinical diagnosis does not necessarily confirm the underlying cause of disease. We also note that logistic regression removes cases list-wise, reducing the power of the analysis. Because of the nature of neurodegenerative diseases, some patients were too severely impaired to complete all assessments. Missing variables resulted in the removal of 24 patients from the analysis, despite some recorded behavioral changes, demographics, and diagnosis. Although methods such as multiple imputation can be used to estimate scores based on other available measures, they are uncommon in logistic regression studies, and are not without assumptions that would be difficult to justify in our study (e.g., that missing data are missing at random). It is also possible that our cohort is biased or unrepresentative of the full spectrum of disorders associated with FTLD, although we sought to minimize such biases by the multiplicity of case ascertainment methods. We do not perform subanalyses by race, as $>95 \%$ of the PiPPIN region patients are classed as "white Caucasians." This prevents our examination of potential racial differences in disease expression and prognosis.

Apathy and related functional impairment in FTLD syndromes effectively predicts mortality. The prognostic importance of these neurobehavioral features may provide a means to effectively predict survival and stratify patients for clinical trials, for example into apathetic (rapid progressor) and nonapathetic (slow progressor) groups. Identification and enrollment of patients at greater risk of disease progression would maximize power to detect a therapeutic effect in forthcoming clinical trials. The prognostic importance of apathy highlights the need to develop more effective and targeted measurement tools to improve recognition and provide outcome measures for clinical studies. Indeed, effective symptomatic interventions targeting the neurobiology of apathy might even improve prognosis.

\section{Author contributions}

C.J. Lansdall and J.B. Rowe are the primary authors. J.B. Rowe, I.T.S. Coyle-Gilchrist, and T.W. Robbins were responsible for study design. C.J. Lansdall undertook statistical analyses. I.T.S. Coyle-Gilchrist, C.J. Lansdall, P. Vázquez Rodríguez, A. Wilcox, E. Wehmann, and J.B. Rowe were responsible for patient data collection. All authors contributed to manuscript preparation.

\section{Acknowledgment}

The authors acknowledge the support of all the patients and their relatives who agreed to participate in this study, the PSP Association (UK) and FTD Support Group (UK) for promoting the study, and the NIHR Biomedical Research Centre's Cambridge Brain Bank. 


\section{Study funding}

The study was funded by the NIHR Cambridge Biomedical Research Centre, Cambridge Home and EU Scholarship Scheme, James F. McDonnell Foundation, Wellcome Trust (103838), Medical Research Council, and Evelyn Trust.

\section{Disclosure}

C. Lansdall, I. Coyle-Gilchrist, P. Vázquez Rodríguez, A. Wilcox and E. Wehmann report no disclosures relevant to the manuscript. T. Robbins: consultancy for Cambridge Cognition, Lundbeck, Mundipharma and Otsuka; research grants from Lundbeck and Shionogi; Royalties for CANTAB from Cambridge Cognition; and editorial honoraria from Psychopharmacology (Springer) and Current Opinion in the Behavioral Sciences (Elsevier). J. Rowe: consultancy for Asceneuron; research grants from Janssen, AZ-Medimmune; and serves as editor for Brain. Go to Neurology.org/N for full disclosures.

\section{Publication history}

Received by Neurology June 14, 2018. Accepted in final form November 21, 2018.

\section{References}

1. Chiu WZ, Kaat LD, Seelaar H, et al. Survival in progressive supranuclear palsy and frontotemporal dementia. J Neurol Neurosurg Psychiatry 2010;81:441-445.

2. Coyle-Gilchrist ITS, Dick KM, Patterson K, et al. Prevalence, characteristics, and survival of frontotemporal lobar degeneration syndromes. Neurology 2016;86: 1736-1743.

3. Roberson ED, Hesse JH, Rose KD, et al. Frontotemporal dementia progresses to death faster than Alzheimer disease. Neurology 2005;65:719-725.

4. Jecmenica-Lukic M, Petrovic IN, Pekmezovic T, Kostic VS. Clinical outcomes of two main variants of progressive supranuclear palsy and multiple system atrophy: a prospective natural history study. J Neurol 2014;261:1575-1583.

5. Hodges JR, Davies R, Xuereb J, Kril J, Halliday G. Survival in frontotemporal dementia. Neurology 2003;61:349-354.

6. Borroni B, Grassi M, Agosti C, et al. Survival in frontotemporal lobar degeneration and related disorders: latent class predictors and brain functional correlates. Rejuvenation Res 2009;12:33-44.

7. Garcin B, Lillo P, Hornberger M, et al. Of survival in behavioral variant frontotemporal dementia. Neurology 2009;73:1656-1661.

8. Xie SX, Forman MS, Farmer J, et al. Factors associated with survival probability in autopsy-proven frontotemporal lobar degeneration. J Neurol Neurosurg Psychiatry 2008;79:126-129.

9. Höglinger GU, Respondek G, Stamelou M, et al. Clinical diagnosis of progressive supranuclear palsy: the Movement Disorder Society criteria. Mov Disord 2017;32: 853-864.

10. Lansdall CJ, Coyle-Gilchrist ITS, Jones PS, et al. Apathy and impulsivity in frontotemporal lobar degeneration syndromes. Brain 2017;140:1792-1807.

11. Hollocks MJ, Lawrence AJ, Brookes RL, et al. Differential relationships between apathy and depression with white matter microstructural changes and functional outcomes. Brain 2015;138:3803-3815.

12. Paulsen JS, Ready RE, Hamilton JM, Mega MS, Cummings JL. Neuropsychiatric aspects of Huntington's disease. J Neurol Neurosurg Psychiatry 2001;5:310-314.

13. Dujardin K, Sockeel P, Delliaux M, Destée A, Defebvre L. Apathy may herald cognitive decline and dementia in Parkinson's disease. Mov Disord 2009;24:2391-2397.
14. Kertesz A, McMonagle P, Jesso S. Extrapyramidal syndromes in frontotemporal degeneration. J Mol Neurosci 2011;45:336-342.

15. Merrilees J, Dowling G, Hubbard E, Mastick J, Ketelle R, Miller B. Characterization of apathy in persons with frontotemporal dementia and the impact on family caregivers. Alzheimer Dis Assoc Disord 2014;27:62-67.

16. O'Connor CM, Landin-Romero R, Clemson L, et al. Behavioral-variant frontotemporal dementia: distinct phenotypes with unique functional profiles. Neurology 2017;89:570-577.

17. Robert P, Onyike CU, Leentjens AF, et al. Proposed diagnostic criteria for apathy in Alzheimer's disease and other neuropsychiatric disorders. Eur Psychiatry 2009;24: 98-104.

18. Caga J, Turner MR, Hsieh S, et al. Apathy is associated with poor prognosis in amyotrophic lateral sclerosis. Eur J Neurol 2016;23:891-897.

19. Tun S, Daniel L, Heidi L, Christopher C, von Eye A. Predictive validity of neuropsychiatric subgroups on nursing home placement and survival in patients with Alzheimer disease. Am J Geriatr Psychiatry 2007;15:314-327.

20. Wenning GK, Litvan I, Jankovic J, et al. Natural history and survival of 14 patients with corticobasal degeneration confirmed at postmortem examination. J Neurol Neurosurg Psychiatry 1998;64:184-189.

21. Mioshi E, Dawson K, Mitchell J, Arnold R, Hodges JR. The Addenbrooke's Cognitive Examination Revised (ACE-R): a brief cognitive test battery for dementia screening. Int J Geriatr Psychiatry 2006;21:1078-1085.

22. Marin RS, Biedrzycki RC, Firinciogullari S. Reliability and validity of the Apathy Evaluation Scale. Psychiatry Res 1991;38:143-162.

23. Firinciogullari J, Mega M, Gray K, Rosenberg-Thompson S, Carusi D, Gornbein J. The Neuropsychiatric Inventory: comprehensive assessment of psychopathology in dementia. Neurology 1994;44:2308-2314.

24. Wear HJ, Wedderburn CJ, Mioshi E, et al. The Cambridge Behavioural Inventory Revised. Dement Neuropsychol 2008;2:102-107.

25. Hu WT, Shelnutt M, Wilson A, et al. Behavior matters-cognitive predictors of survival in amyotrophic lateral sclerosis. PLoS One 2013;8:e57584.

26. Borroni B, Grassi M, Agosti C, et al. Latent profile analysis in frontotemporal lobar degeneration and related disorders: clinical presentation and SPECT functional correlates. BMC Neurol 2007;7:9.

27. Lansdall CJ, Coyle-Gilchrist ITS, Jones PS, et al. White matter change with apathy and impulsivity in frontotemporal lobar degeneration syndromes. Neurology 2018;90: e1066-e1076.

28. Josephs KA, Petersen RC, Knopman DS, et al. Clinicopathologic analysis of frontotemporal and corticobasal degenerations and PSP. Neurology 2006;66:41-48.

29. Robert PH, Berr C, Volteau M, et al. Importance of lack of interest in patients with mild cognitive impairment. Am J Geriatr Psychiatry 2008;16:770-776.

30. Josephs K, Knopman D, Whitwell J, et al. Survival in two variants of tau-negative frontotemporal lobar degeneration: FTLD-U vs FTLD-MND. Neurology 2005;65: 645-647.

31. Kertesz A, Hillis A, Munoz DG. Frontotemporal degeneration, Pick's disease, Pick complex, and Ravel. Ann Neurol 2003;54(suppl 5):S1-S2.

32. Cuthbert BN. Research Domain Criteria: toward future psychiatric nosologies. Dialogues Clin Neurosci 2015; 17:89-97.

33. Rittman T, Coyle-Gilchrist ITS, Rowe JB. Managing cognition in progressive supranuclear palsy. Neurodegen Dis Manag 2016;6:499-508.

34. Ghosh BCP, Calder AJ, Peers PV, et al. Social cognitive deficits and their neural correlates in progressive supranuclear palsy. Brain 2012;135:2089-2102.

35. Armstrong MJ, Litvan I, Lang AE, et al. Criteria for the diagnosis of corticobasal degeneration. Neurology 2013;80:496-503.

36. Litvan I, Mega MS, Cummings JL, Fairbanks L. Neuropsychiatric aspects of progressive supranuclear palsy. Neurology 1996;47:1184-1189.

37. Litvan I, Cummings JL, Mega M. Neuropsychiatric features of corticobasal degeneration. J Neurol Neurosurg Psychiatry 1998;65:717-721.

38. Murley AG, Rowe JB. Neurotransmitter deficits from frontotemporal lobar degeneration. Brain 2018;141:1263-1285.

39. Alexander SK, Rittman T, Xuereb JH, Bak TH, Hodges JR, Rowe JB. Validation of the new consensus criteria for the diagnosis of corticobasal degeneration. J Neurol Neurosurg Psychiatry 2014;85:923-927.

40. Dubois B, Slachevsky A, Litvan I, Pillon B. The FAB: a Frontal Assessment Battery at bedside. Neurology 2000;55:1621-1626. 


\section{Neurology}

\section{Prognostic importance of apathy in syndromes associated with frontotemporal lobar degeneration}

Claire J. Lansdall, Ian T.S. Coyle-Gilchrist, Patricia Vázquez Rodríguez, et al. Neurology 2019;92;e1547-e1557 Published Online before print March 6, 2019

DOI 10.1212/WNL.0000000000007249

\section{This information is current as of March 6, 2019}

\section{Updated Information \&} Services

References

Citations

Subspecialty Collections

Permissions \& Licensing

Reprints including high resolution figures, can be found at: http://n.neurology.org/content/92/14/e1547.full

This article cites 40 articles, 16 of which you can access for free at: http://n.neurology.org/content/92/14/e1547.full\#ref-list-1

This article has been cited by 1 HighWire-hosted articles: http://n.neurology.org/content/92/14/e1547.full\#\#otherarticles

This article, along with others on similar topics, appears in the following collection(s):

Corticobasal degeneration

http://n.neurology.org/cgi/collection/corticobasal_degeneration

Frontotemporal dementia

http://n.neurology.org/cgi/collection/frontotemporal_dementia

Progressive supranuclear palsy

http://n.neurology.org/cgi/collection/progressive_supranuclear_palsy

Information about reproducing this article in parts (figures,tables) or in its entirety can be found online at:

http://www.neurology.org/about/about_the_journal\#permissions

Information about ordering reprints can be found online:

http://n.neurology.org/subscribers/advertise

Neurology ${ }^{\circledR}$ is the official journal of the American Academy of Neurology. Published continuously since 1951, it is now a weekly with 48 issues per year. Copyright Copyright ( 2019 The Author(s). Published by Wolters Kluwer Health, Inc. on behalf of the American Academy of Neurology.. All rights reserved. Print ISSN: 0028-3878. Online ISSN: 1526-632X.

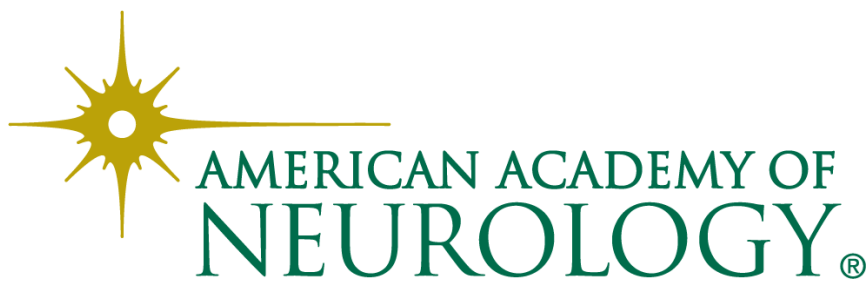

